\title{
Les analyses
}

\section{Les migrations internationales et l'Afrique : des logiques Sud-Nord ou Sud-Sud?}

\author{
par
}

le recteur Gérard-François DUMONT

Professeur à Sorbonne-Université

Président de la revue Population \& Avenir 


\section{Les migrations internationales et l'Afrique : des logiques Sud-Nord ou Sud-Sud?}

Gérard-François Dumont

\section{Résumé}

Les migrations internationales concernant l'Afrique couvrent deux champs géographiques. Le premier, Sud-Nord, s'explique notamment par le souci de populations d'améliorer leurs conditions de vie et par des liens historiques, dans un contexte où la gouvernance des pays africains engendre diverses difficultés de développement. Le second concerne les migrations intracontinentales africaines Sud-Sud, dont les facteurs doivent être analysés.

\section{Mots-clés}

Migrations ; migrations internationales ; Afrique ; population ; gouvernance ; développement; Europe ; Amérique du Nord ; Égypte ; Sénégal ; Ouganda ; Maroc ; Tchad ; Darfour

\section{Abstract}

International migrations for Africa cover two geographical areas. The first, South-North, can be explained by the desire of people to improve their living conditions and by historical links, in a context where the governance of African countries create many development challenges. The second concerns the African intra-continental migrations South-South whose analysis can lead to highlight nature.

\section{Keywords}

Migrations ; international migration; Africa; population; governance ; development; Europe; North America ; Egypt ; Senegal; Uganda; Morocco ; Chad; Darfur

Pour citer cette publication :

To cite this version :

Dumont, Gérard-François, « Les migrations internationales et l'Afrique : des logiques Sud-Nord ou Sud-Sud? ", Les analyses de Population \& Avenir, mars 2019, p. 117. [ISSN 2552-2078] 


\section{Sommaire}

UN CONTINENT D'EMIGRATION

L'AFRIQUE, UN CONTINENT GLOBALEMENT REPULSIF ……............................................................... 5

LES FACTEURS DE L'ATTRACTIVITE D'AUTRES CONTINENTS POUR LES AFRICAINS .............................. 7

DES SYSTEMES MIGRATOIRES FORT DIVERSIFIES SELON LES PAYS AFRICAINS......11

LA GEOGRAPHIE VARIEE ET MOUVANTE DES FACTEURS DE REPULSION .............................................11

LA DIVERSITE DES FACTEURS D'ATTRACTION ………….............................................................. 
La réponse à la question posée est simple. Les migrations internationales concernant l'Afrique couvrent effectivement deux champs géographiques principaux, Sud-Nord et Sud-Sud, même s'il existe des migrations Nord-Sud. Le premier s'explique notamment par le souci de populations d'améliorer leurs conditions de vie et par des liens historiques, dans un contexte où la gouvernance des pays africains engendre diverses difficultés de développement. Le second concerne les migrations intracontinentales africaines, donc Sud-Sud ${ }^{1}$, dont les facteurs doivent être analysés.

Il faut toutefois préciser que le premier problème soulevé par toute analyse des migrations concerne l'insuffisance des statistiques en ce domaine. Par exemple, les États africains ne possèdent pas, tout comme la France, d'ailleurs, de système d'information complet sur les flux de migrations permettant de distinguer les flux d'émigration et ceux d'immigration. Il n'est donc pas aisé d'indiquer des chiffres à la fiabilité certaine sur les migrations internationales concernant l'Afrique ${ }^{2}$. Les statistiques livrées, qui portent essentiellement sur des soldes migratoires, doivent être examinées avec sens critique vu qu'il s'agit d'estimations brutes établies en méconnaissance des données nettes.

Dans ce contexte statistique insatisfaisant, il convient donc moins de vouloir analyser une quantification, d'ailleurs imprécise, des migrations que de comprendre, d'une part, la nature du système migratoire du continent africain et, d'autre part, les spécificités des systèmes migratoires propres à chaque pays de ce continent ${ }^{3}$.

\footnotetext{
${ }_{2}^{1}$ Auxquelles s'ajoutent d'autres migrations, comme celle concernant les Chinois.

${ }^{2}$ Prenons un seul exemple d'un territoire africain qui est une région ultrapériphérique de l'Union européenne : Mayotte. Ce territoire, qui a le statut de département français depuis 2011, connaît, en 2015, un taux d'accroissement migratoire de -5 pour mille habitants selon le PRB, de $-1,3$ selon le WPDS (pour la période 2010-2015) et de 0 selon l'Insee. Tous ces chiffres sont discutables car il est en fait fort probable que Mayotte a un taux d'accroissement migratoire positif, les départs de Mahorais vers La Réunion et la métropole étant certainement compensés par des arrivées d'Anjouanais et de natifs d'autres départements français, sachant d'autre part que la population de Mayotte est estimée comme étant composée à $29 \%$ d'immigrants nés à l'étranger: 61000 en 2012 (chiffre considéré comme sous-évalué compte tenu de l'importance des personnes en situation illégale) sur une population totale de 213000 habitants; cf. Insee première, $n^{\circ} 1468$, février 2014.

${ }^{3} \mathrm{Au}$ regard de la question plus générale des migrations internationales ; cf. Dumont, Gérard-François, Géographie des populations. Concepts, dynamiques, prospectives, Paris, Armand Colin, 2018.
} 


\section{Un continent d"émigration}

La migration internationale africaine est certes actuellement très dynamique, mais elle est encore faible par rapport aux autres continents. En 2017, le pourcentage des immigrants africains dans le total des immigrants dans le monde - 257,7 millions ${ }^{4}$ est de $14,1 \%$ contre $41 \%$ pour l'Asie, $23,7 \%$ pour l'Europe et $14,6 \%$ pour l'Amérique latine et Caraïbe 5 .

Table continents (en milliers)

\begin{tabular}{|lr|r|r|r|}
\cline { 2 - 5 } \multicolumn{1}{c|}{} & Population & $\begin{array}{c}\text { \% par } \\
\text { rapport à la } \\
\text { population dans le } \\
\text { monde }\end{array}$ & $\begin{array}{r}\text { Nombre } \\
\text { d'immigrants } \\
\text { originaires }\end{array}$ & $\begin{array}{c}\text { \% du total } \\
\text { des immigrants } \\
\text { internationaux }\end{array}$ \\
\hline Monde & 7550262 & 100 & 257715 & 100,0 \\
\hline Afrique & 1256268 & 16,6 & 36266 & 14,1 \\
\hline Asie & 4504428 & 59,7 & 105684 & 41,0 \\
\hline Europe & 742074 & 9,8 & 61191 & 23,7 \\
\hline Amérique latine et Caraïbes & 645593 & 8,6 & 37720 & 14,6 \\
\hline Amérique du Nord & 361208 & 4,8 & 4413 & 1,7 \\
\hline Océanie & 40691 & 0,5 & 1880 & 0,7 \\
\hline Origine inconnue & - & - & 10560 & 4,1 \\
\hline
\end{tabular}

Chiffres ONU (2017).

L'Afrique, considérée dans son ensemble, est un continent structurellement répulsif. Selon les données de la Division de la population de l'ONU, son taux d'accroissement migratoire est constamment négatif depuis 1950, sauf, au début des années 1990, au lendemain de l'éclatement de l’Union Soviétique. Ce taux d'accroissement migratoire signifie un excédent des départs du continent sur les arrivées qui varie selon les années autour d'un chiffre de 2 millions, que l'ONU estime plus élevé, à 2,9 millions par an pour la période 2010-20156. La nature de l'Afrique en tant que continent d'émigration tient à des facteurs de répulsion et d'attraction.

\section{L"Afrique, un continent globalement répulsif}

L'émigration est, pour les Africains, une façon de "voter avec leurs pieds », de signifier une double insatisfaction. La première est une insatisfaction politique résultant de l'incapacité de trop de dirigeants africains à œuvrer pour le bien commun et la concorde sociale dans leurs pays ainsi que des insuffisances démocratiques mises

\footnotetext{
${ }^{4}$ Contre 172,6 millions en 2000.

${ }^{5}$ United Nations, International migration report, 2017, p. 9

${ }^{6}$ WPDS, The 2015 revision.
} 
en évidence par des méthodes autoritaires ou un contrôle des élections qui ne permet pas toujours de considérer leurs résultats comme fiables. La seconde insatisfaction tient à cette contradiction entre les richesses considérables du sol comme du sous-sol africains et un niveau de développement qui demeure, par exemple au regard du PIB par habitant, nettement inférieur à celui de tous les autres continents ou souscontinents, donc inférieur aussi au sous-continent non africain le plus pauvre, l'Asie centrale du Sud.

Autrement dit, l'émigration africaine s'explique par les difficultés que rencontrent les États de ce continent - dont l'indépendance date, pour la majorité d'entre eux, des années 1950 et 1960 - dans la mise en œuvre d'un système politique facilitant le développement. Dans ces conditions, un nombre croissant des jeunes actifs ne parvient pas « à vivre et travailler au pays », en raison des rigidités économiques, des privilèges des nomenklaturas, des contraintes de la corruption ou de l'étouffement de l'initiative économique par des procédures bureaucratiques.

Les deux insatisfactions précisées ci-dessus peuvent être complétées périodiquement par une troisième, lors de périodes marquées par de la violence politique. Les exemples en sont nombreux : émigration des Ougandais originaires du sous-continent indien ${ }^{7}$ vers le Royaume-Uni, lors de leur expulsion par Idi Amin Dada en 1972 ; émigration algérienne accentuée vers la France lors de la guerre civile algérienne des années 1990 entre le pouvoir militaire et les islamistes ; émigration accrue des Coptes, notamment vers l'Amérique du Nord, lors de l'accès à la présidence de l'Égypte par un frère musulman, de juin 2012 à juillet 2013, etc.

L'importance de l'émigration africaine peut également s'expliquer par des effets d'âge résultant de la composition par âge très jeune des populations africaines ${ }^{8}$. En effet, l'histoire migratoire enseigne que la propension à migrer est assez bien corrélée avec l'âge, avec une plus forte intensité dans la tranche d'âge 18-30 ans. Or, compte tenu de la dynamique démographique de la population africaine de ces dernières décennies, les générations qui parviennent dans cette tranche d'âge sont de plus en plus nombreuses. Il faut également noter que les pays africains enregistrent les indices de fécondité parmi les plus élevées au monde ${ }^{9}$ alors que ces taux sont, en général, inversement proportionnels au PIB par habitant et de taux d'accès à l'électricité. Les taux élevés de fécondité dans le continent n'ont pas encore des incidences économiques suffisamment favorables pour le continent ${ }^{10}$. Donc, même si le taux d'émigration ${ }^{11}$ était faible, les résultats pourraient se traduire par des flux importants.

${ }^{7}$ Environ 50000 furent expulsés, en majorité vers le Royaume-Uni car l'Inde s'est alors montré fort peu accueillante dans cette période où elle se désintéressait de ses diasporas. Cf. Dumont, Gérard-François, "L'Inde et sa diaspora : une synergie géopolitique », dans: Pierre Verluise (direction), Histoire, Géographie et Géopolitique de l'Asie, Éditions Diploweb via Amazon, 2018.

${ }^{8}$ Dumont, Gérard-François, «La population de l'Afrique », dans: Frérot, Anne-Marie (direction), L'Afrique en questions, Paris, Ellipses, 2004

${ }^{9}$ Sardon, Jean-Paul, «La population des continents et des pays », Population \& Avenir, $\mathrm{n}^{\circ} 740$, novembre-décembre 2018.

${ }^{10}$ Sène, Abdourahmane Mbade, «Afrique : évolution de la fécondité et enjeux de développement ", Population \& Avenir, $n^{\circ} 735$, novembre-décembre 2017.

${ }^{11}$ En réalité, ce taux n'est pas disponible, tout comme d'ailleurs en France, compte tenu des insuffisances du système statistique. En revanche, puisque l'estimation du taux d'accroissement migratoire donne un résultat négatif, le taux d'émigration est nécessairement supérieur au taux d'immigration. 
L'émigration africaine s'explique non seulement par des facteurs répulsifs mais aussi parce que d'autres continents semblent attractifs.

\section{Les facteurs de l'attractivité d'autres continents pour les Africains}

La première région du monde attractive aux yeux des Africains est l'Europe dans sa partie ouest car celle-ci est la plus proche, géographiquement et historiquement, de l'Afrique ${ }^{12}$. Cette attractivité résulte en fait d'une combinaison de facteurs d'attraction.

D'abord, comme héritage de la colonisation, l'Europe de l'Ouest a laissé à l'Afrique ses langues. C'est pourquoi de nombreux pays d'Afrique partagent avec un pays d'Europe une même langue, française, anglaise, portugaise ou espagnole, qui continue le plus souvent d'être enseignée et utilisée, voire constitue parfois l'unique langue officielle. L'Africain qui, venant de pays où la pratique d'une langue européenne est courante, se retrouve immigrant dans un pays européen qui est la métropole de l'ancienne puissance coloniale, ne subit pas de dépaysement linguistique, ce qui limite ses difficultés d'intégration.

Cette attractivité linguistique se trouve complétée par les législations nationales des pays européens ou les conventions internationales qu'ils ont signées. En effet, les avancées démocratiques des pays européens les ont conduits à développer l'octroi de droits aux immigrants. Cela concerne d'abord le droit d'entrée dans le pays à travers des législations nationales ou l'application de la Convention européenne des droits de l'homme prévoyant le droit au regroupement familial dans son article 8, ou la Convention de Genève sur le droit d'asile. En outre, les émigrants africains savent que, s'ils choisissent l'Europe comme destination, ils y bénéficient de droits d'association, syndicaux ou même politiques. Par exemple, ils savent que des étrangers peuvent librement créer des associations en France, qu'ils sont électeurs aux instances de représentation des salariés dans les entreprises ou qu'ils ont, au Royaume-Uni, le statut de citoyen s'ils sont ressortissants d'un pays du Commonwealth, ce qui leur donne par exemple le droit de vote aux élections parlementaires britanniques.

\footnotetext{
12 En 2017, selon les estimations des Nations Unies, l'Europe a accueilli 9 millions d'immigrants africains ; cf. United Nations, International migration report, 2017, p. 11..
} 
Figure 1. Nombre d'immigrants (en continent

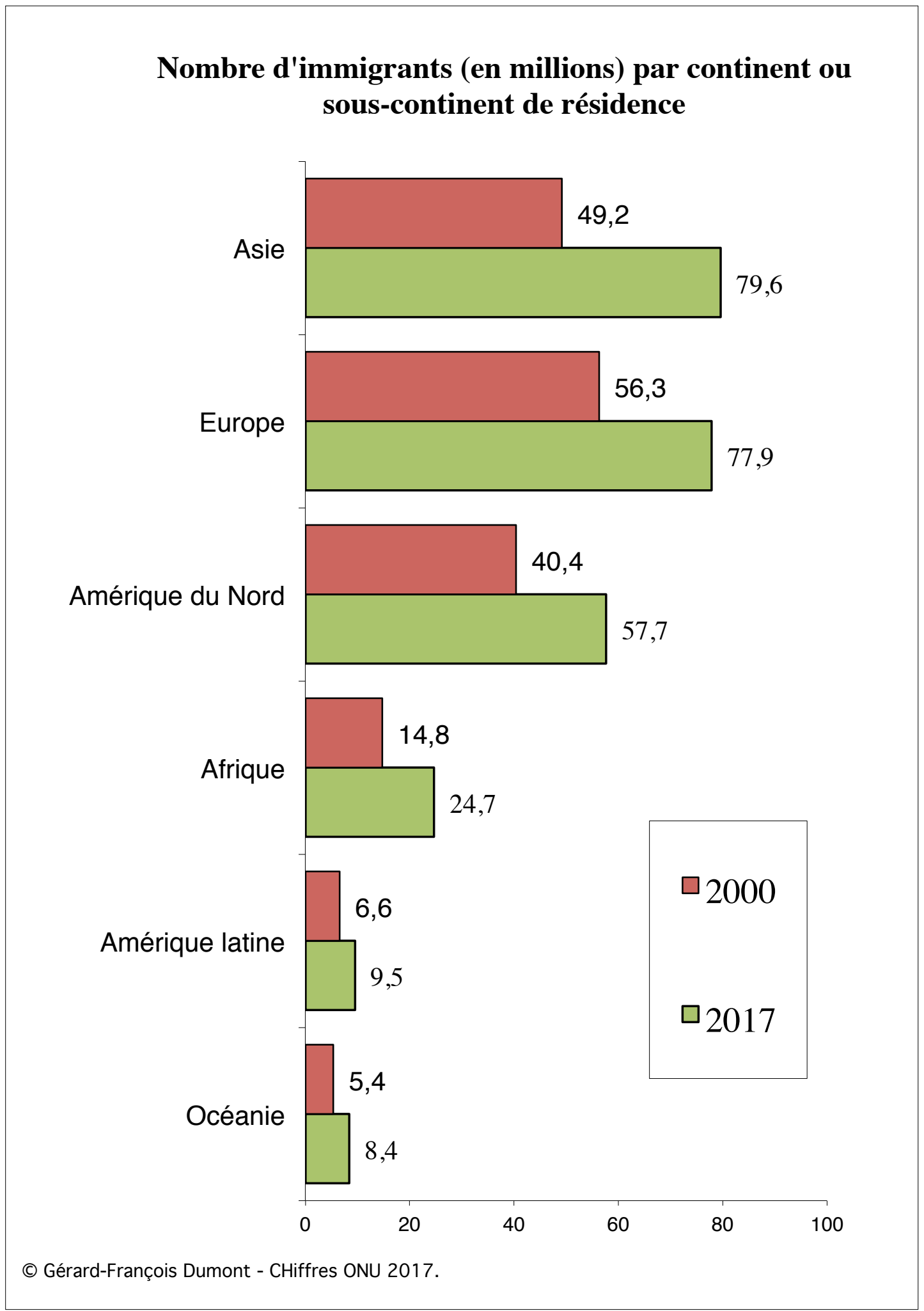

Note de lecture : en 2017, parmi les 257,7 millions de personnes qui habitent un autre pays que celui où elles sont nées, 24,7 millions résident en Afrique. Il y en avait 14,8 millions en 2000. 
Figure 1. Nombre d'immigrants (en millions) par_continent on ou souscontinnent

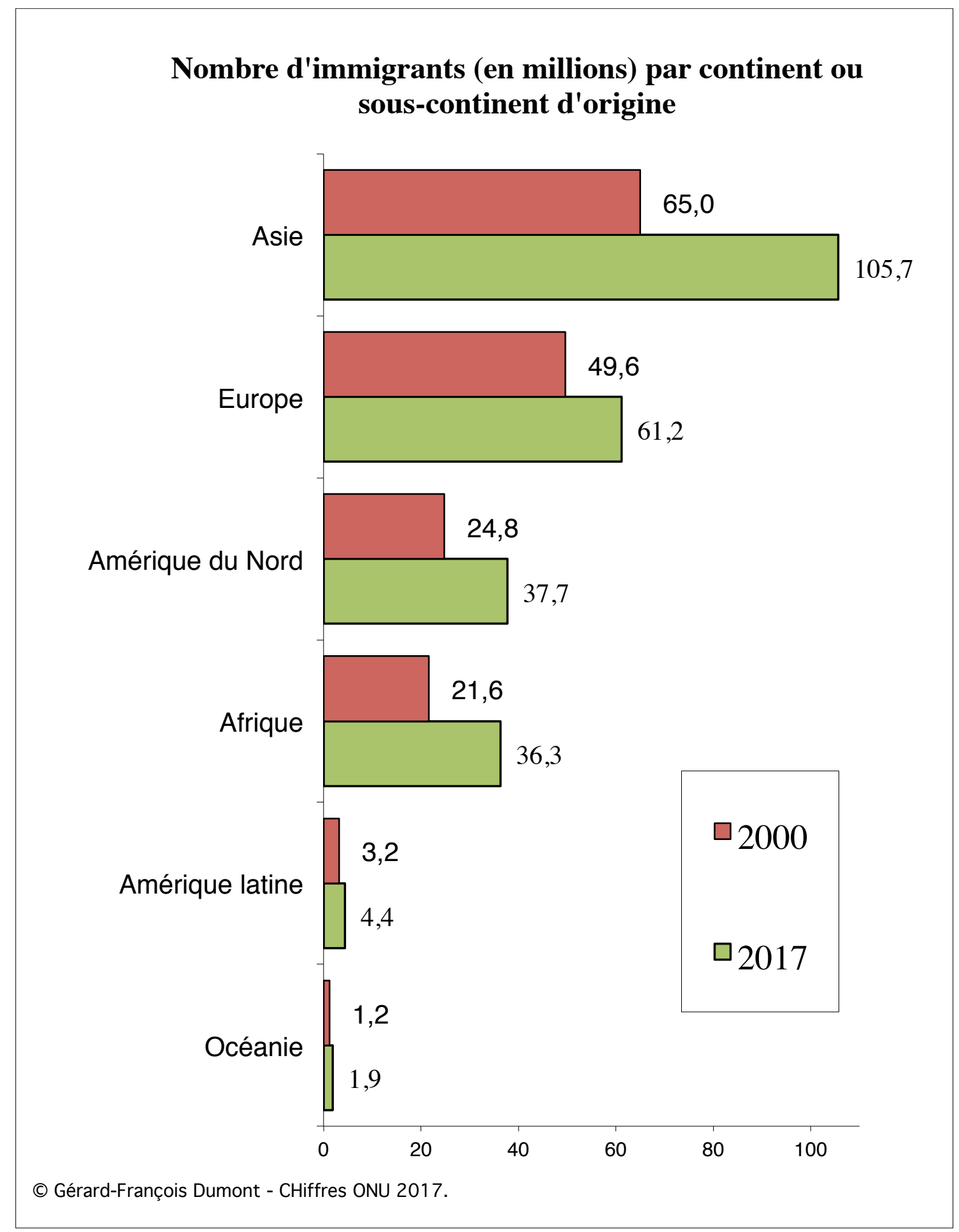

Note de lecture : en 2017, parmi les 247,2 millions de personnes qui habitent un autre pays que celui où elles sont nées, et dont on connaît précisément le pays du lieu de naissance, 36,3 millions sont nées en Afrique. Il y en avait 21,6 millions en 2000. 
Les émigrants savent également les assez larges possibilités dont ils disposent pour acquérir la nationalité d'un pays européen et, surtout, pour l'acquérir sans devoir renoncer à la nationalité de leur pays d'origine. Le droit à pouvoir disposer de deux passeports est donc un facteur supplémentaire d'attractivité de plusieurs pays européens. Les Africains savent aussi que les besoins de l'Europe en migrations de remplacement ${ }^{13}$ sont élevés. D’une part, certains pays européens connaissent une diminution de leur population active après plusieurs décennies de ce que j'ai appelé "l'hiver démographique», c'est-à-dire une fécondité nettement et durablement inférieure au seuil de simple remplacement des générations. D’autre part, nombre d'entreprises européennes souhaitent faire appel à de la main-d'œuvre immigrée en raison de la faible appétence de nationaux pour certains métiers dans le bâtiment, la restauration ou les métiers de la bouche par exemple.

Quant aux Africains immigrants en Europe qui se trouvent en situation illégale, parce qu'ils y sont restés après l'échéance d'une autorisation de séjour, ou parce qu'ils s'y sont rendus par des voies illégales, ils savent que les pays européens procèdent régulièrement à des régularisations d'immigrés clandestins. Et une mesure de régularisation ouvre le droit au regroupement familial. En outre, les télévisions renforcent le « rêve européen » de jeunes actifs africains tandis que la présence accrue de ressortissants d'une nationalité ou d'une ethnie donnée ${ }^{14}$ dans telle ou telle région européenne concourt à faciliter l'accueil de nouveaux ressortissants de cette nationalité.

Tous ces facteurs d'attraction vers l'Europe ne signifient pas que l'Europe soit pour l'Afrique la seule région attractive. En effet, tous les autres continents le sont également pour les Africains, tout simplement parce qu'ils sont tous plus riches. Mais l'intensité de leur caractère attractif est variable. Ainsi, les Africains ont moins d'appétence, par exemple, pour les pays du Golfe. Certes, certains y vont car ils peuvent $\mathrm{y}$ trouver du travail et y améliorer leurs revenus, mais ils savent que leurs droits dans ces pays sont extrêmement réduits et les conditions de travail souvent très dures. De son côté, l'Asie orientale est devenue attirante, surtout depuis l'ouverture économique de la Chine. Quant à l'Amérique du Nord ou à l'Amérique latine, elles le sont de plus en plus compte tenu de la forte baisse du coût de la migration vers ce continent et de la diminution considérable des inconvénients dus à la distance, avec l'essor du processus que j'ai dénommé « internationalisation » ${ }^{15}$. Ce processus se traduit par des transports aériens plus rapides ${ }^{16}$ et moins chers comme par la quasi-instantanéité de la transmission des informations, le développement de la téléphonie mobile et de l'Internet facilitant l'essor de réseaux migratoires à longue distance.

13 Dumont, Gérard-François, «Afrique-Europe : l'inversion démographique », dans : Cluzel, Jean, Solidarité Europe-Afrique, Paris, Economica, octobre 2013.

${ }^{14}$ Comme les Soninkés; cf. N'Diaye, Hamidou, « Une ville africaine en Île-de-France ? Les Soninké entre Montreuil et le Val-Fourré », Thèse de l'Université Paris Ouest, soutenue le 6 février 2014.

${ }^{15}$ Dumont, Gérard-François, "Les nouvelles logiques migratoires », dans: Université de tous les savoirs, sous la direction d'Yves Michaud, Qu'est-ce que la Globalisation ?, Paris, Éditions Odile Jacob, 2004.

${ }^{16}$ Par exemple, le Sénégalais allant aux États-Unis qui devait passer par l'aéroport Paris-Charles de Gaulle dispose depuis 2008 de vols directs Dakar-Atlanta; cf. Kanté Seydou, Géopolitique de l'émigration sénégalaise en France et aux États-Unis, Paris, L'Harmattan, 2014. 
Même si l'Afrique est globalement répulsive, ce serait une erreur d'omettre le fait que ce continent attire aussi des immigrants originaires d'autres continents, il est vrai beaucoup moins nombreux que les émigrants, et entrant généralement dans le cadre d'une migration temporaire. Ses immigrants internationaux sont originaires essentiellement de l'Europe. Il faut toutefois noter que le continent est de plus en plus attractif pour les autres continents comme l'Asie dont le pourcentage d'immigrants résidents en Afrique est passé de $10 \%$ en 2010 à $12 \%$ en 2017 et l'Amérique du Nord, avec un pourcentage d'immigrants en Afrique qui passe de $5 \%$ en 2010 à $7 \%$ en 2017. En revanche, pour l'Europe, le pourcentage d'immigrants en Afrique est resté constant à $26 \%$ entre 2010 et $2017^{17}$. En effet, compte tenu de son potentiel de richesses, l'Afrique attire ce que j'ai appelé les migrations entrepreneuriales ${ }^{18}$, c'est-à-dire «des migrations liées aux décisions d'entreprises faisant migrer leurs collaborateurs face aux évolutions des marchés ou d'actifs souhaitant bénéficier de territoires leur donnant davantage de satisfaction professionnelle». Ainsi l'Afrique voit-elle arriver des actifs de pays du Nord, souvent salariés de groupes ayant comme stratégie de déployer des activités de production, de commercialisation, de distribution ou de services en Afrique, comme Nestlé, Air liquide, L'Oréal, Lafarge, Total, Bolloré ou Canal plus. S'y ajoutent des migrations de travailleurs non ou peu qualifiés, essentiellement en raison du développement de la puissance chinoise en Afrique.

Globalement, l'Afrique est donc incontestablement un continent d'émigration. Toutefois, les systèmes migratoires des pays africains sont diversifiés selon les pays et peuvent aussi se modifier selon leurs évolutions géopolitiques et économiques.

\section{Des systèmes migratoires fort diversifiés selon les pays africains}

La nature des systèmes migratoires des pays africains varie selon l'intensité des facteurs de répulsion ou d'attraction propres à chaque pays.

\section{La géographie variée et mouvante des facteurs de répulsion}

Les facteurs de répulsion peuvent être de nature politique ou économique. Les raisons politiques explicatives des flux d'émigration africaine relèvent de plusieurs types. La première tient à la mise en œuvre par des autorités politiques d'un génocide, auquel cas des populations parvenant à en réchapper forment autant de rescapés. Ainsi le génocide du Rwanda de 1994 a-t-il vu de nombreux rescapés se réfugier dans les pays voisins de l'Afrique centrale ou de l'Afrique orientale. De même, les violences du gouvernement soudanais au Darfour, parfois qualifiées de génocide, ont poussé des

${ }_{17}^{17}$ United Nations, International migration report, 2017.

${ }^{18}$ Dumont, Gérard-François, Les migrations internationales. Les nouvelles logiques migratoires, Paris, Éditions Sedes, 1995. 
Soudanais à aller se réfugier au Tchad ${ }^{19}$. Les événements qui se déroulent au Burundi, depuis la réélection contestée ${ }^{20} \mathrm{du}$ président à la présidentielle du 21 juillet, n'ont pas été qualifiés de génocide. Mais l'importance des brutalités ${ }^{21}$ s'inscrit dans un cycle de violences qui poussent des populations à passer la frontière pour se réfugier essentiellement dans les pays limitrophes du Burundi.

Le deuxième type d'émigration politique apparaît chaque fois qu'une guerre, le plus souvent civile, se déclenche sur un territoire africain. Pour assurer leur survie, les populations partent vers le premier territoire où un minimum de sécurité peut être assuré, donc un territoire adjacent à celui de leur pays. Ce mouvement est facilité par l'homogénéité ethnoculturelle souvent notée de part et d'autre des frontières africaines et leur perméabilité. Par exemple, en Casamance au sud du Sénégal, pendant les périodes de crise politique, des déplacements de réfugiés se font vers les villages gambiens les plus proches de la frontière servant aux populations de zones de transitions, ensuite vers celles les plus «sécurisées» servant de zones d'accueil, notamment les aires urbaines de Banjul, la capitale de la Gambie ${ }^{22}$. Ainsi, de nombreux flux migratoires intra-africains s'expliquent-ils par les guerres civiles qu'ont connues ou que connaissent certains pays comme l'Angola, le Liberia, le Mozambique, le Sierra Leone, la Somalie ou le Soudan.

En troisième lieu, chaque fois qu'un régime politique met en œuvre des mesures particulièrement liberticides, usant parfois de moyens violents vis-à-vis des populations qui ne lui sont pas favorables, une forte émigration se déploie. Cela a été le cas à certaines périodes au Zimbabwe et l'est toujours en Érythrée, où un régime autoritaire fait subir à la population des contraintes difficilement supportables. Parfois, le régime s'avère particulièrement liberticide vis-à-vis de tel ou tel groupe humain minoritaire, auquel cas certains de ses membres s'estiment contraints à émigrer.

En outre, l'Afrique peut connaître des conflits interethniques à l'origine de migrations. Par exemple, en Afrique occidentale, les différends entre Mauritanie et Sénégal ont également entraîné des flux réciproques, particulièrement en juin 1989, après les "événements"23.

S'ajoutent périodiquement des expulsions politiques liées en fait à une mauvaise gouvernance. Ainsi, des pays connaissant des difficultés économiques n’hésitent-ils pas à renvoyer les travailleurs étrangers dès qu'ils considèrent que leur présence n'est plus indispensable. L'histoire africaine offre de multiples exemples de renvoi de travailleurs étrangers dans une phase économique dépressive. En 1969 le Ghana, essuyant un gros échec en raison de choix économiques erronés, a renvoyé environ 100000 personnes dans leur pays : Haute-Volta, Niger, Bénin, Togo. Les expulsions organisées par le Zaïre

${ }^{19}$ Dumont, Gérard-François, «Le Tchad: diversité, fractures et freins au développement », dans : Dumortier, Brigitte (direction), L'Afrique du Sahel et du Sahara à la Méditerranée, Paris, Armand Colin, 2017.

${ }^{20}$ Cette présidentielle a été boycottée par une large partie de l'opposition, qui estime que ce troisième mandat consécutif de Pierre Nkurunziza, déjà élu à la tête de l'État en 2005 et en 2010, est contraire à la Constitution.

${ }^{21}$ Par exemple Le Monde titre : «Au Burundi, assassinats en série à la tête de l'armée. Bujumbura est plongée dans un climat de terreur, un an après la début de la crise politique », 28 avril 2016, p. 5.

22 Sène, Abdourahmane Mbade, " Impacts du conflit casamançais dans la zone frontalière de la région de Ziguinchor avec la Gambie », Conférence PICARD, Malatya (Turquie), 9-11 octobre 2018.

${ }^{23}$ Le nombre de rapatriés mauritaniens, à la suite de ces heurts, est estimé à 240000 . Quant au Sénégal, le gouvernement a alors indiqué les chiffres de 70229 rapatriés sénégalais et de 45203 réfugiés. 
en 1971 et 1972 ont été justifiées par des "crimes économiques", comme si les travailleurs étrangers étaient responsables du marasme économique : des dizaines de milliers de Sénégalais, de Maliens, de Nigériens et de Mauritaniens ont été reconduits à la frontière. En 1977, c'est au tour du Congo, dont l'économie se dégrade, d'agir de même en refoulant des Sénégalais et des Maliens. Un nombre d'expulsions économiques très important a été constaté au Nigeria à plusieurs reprises. Le "géant de l'Afrique" avait cru que la possession de pétrole était une condition suffisante au développement, ce qui n'est évidemment pas le cas, et cette erreur a mis à mal son économie. Environ deux millions d'étrangers ont dû, dans le premier trimestre 1983, quitter le pays dans des conditions peu honorables, le plus souvent après avoir été dépouillés. Cette émigration de retour a concerné environ un demi-million de Ghanéens, des dizaines de milliers de Camerounais, Béninois, Togolais, Tchadiens ou Burkinabés.

L'émigration liée à des raisons politiques provient toujours du manque d'espoir dû à l'évolution politique du pays. Deux situations politiques opposées le stimulent. D'une part, un pays où la vie politique est marquée par une instabilité chronique ne peut mettre en œuvre des politiques de développement pertinentes car leur réussite suppose qu'elles s'inscrivent dans la durée ; l'insuccès économique étant alors certain, les populations sont poussées à partir. Les Comores, au moins dans les premières décennies de leur indépendance, illustrent ce type, même si d'autres pays africains ont connu une profusion de coups d'État. L'autre type tient, au contraire, à la pérennité abusive d'un pouvoir politique qui parvient à préserver son emprise et, souvent, son caractère prévaricateur sur le pays, empêchant tout renouvellement qui donnerait leur chance à des méthodes innovantes de développement, susceptibles de dynamiser l'économie du pays. Les exemples de manœuvres d'un président sortant pour parvenir à se maintenir au pouvoir ont concerné ou concernent de nombreux pays : CongoBrazzaville, Cameroun, Zimbabwe, etc., même s'il y a eu parfois, mais rarement, des tentatives qui ont échoué, comme au Burkina Faso à l'automne 2014.

Sauf lorsque l'émigration équivaut à un exode rendu impératif pour des populations voulant assurer leur survie, les facteurs politiques répulsifs se combinent souvent avec des facteurs économiques dans la mesure où les politiques conduites par les dirigeants n'induisent pas des espoirs de développement suffisants, notamment lorsque manquent des règles économiques claires et que le niveau de corruption est élevé. Le fait que, en Afrique, les terres appartiennent à l’État exigerait de sa part une excellente gouvernance pour permettre un essor agricole dissuadant les paysans de partir non seulement vers les villes du pays, mais aussi vers l'étranger ${ }^{24}$.

Dans d'autres cas, l'émigration correspond dans une certaine mesure à une « répulsion choisie ». Un premier exemple tient à ce que les frontières résultant de la décolonisation ne correspondent pas nécessairement aux réalités des échanges économiques et leur localisation sur le terrain pas toujours évident. Les disparités politiques et réglementaires entre pays frontaliers se traduisent par des différences

24 Sène, Abdourahmane Mbade, «Aménagement et dégradation des rizières des bas-fonds dans un contexte de changement climatique dans la région de Ziguinchor », Revue Espace Géographique et Société Marocaine, $n^{\circ} 20 / 21$, janvier 2018. 
importantes concernant l'accès aux infrastructures sociales de base et les prix des produits. Les populations des zones frontalières se déplacent pour optimiser les effets de ces inégalités. Ainsi, les migrations transfrontalières des populations contribuent à atténuer les inconvénients nés de l'existence de la frontière et améliorent leurs conditions de vie. En conséquence, les échanges migratoires traditionnels qui existaient avant la création des États actuels et qui s'expliquaient notamment par la géographie et le climat, se perpétuent. Par exemple, entre les territoires du Ghana, du Bénin et du Nigeria, les échanges migratoires ont toujours été intenses, même si la volonté de ces États de devenir maîtres de leurs frontières se traduit périodiquement par une moindre perméabilité des passages. Un second exemple se présente lorsque, dans certains pays africains ou dans certaines régions, l'émigration est devenue une composante sociale permanente, l'habitude ayant été prise de considérer qu'au moins un enfant de la famille doit émigrer pour contribuer aux ressources de la famille. C'est par exemple le cas au Sénégal ${ }^{25}$, pays qui envoie des ressortissants dans de nombreux pays africains mais également hors d'Afrique.

Causées par des facteurs répulsifs, les migrations intra-africaines tiennent aussi à des facteurs d'attraction variés.

\section{La diversité des facteurs d'attraction}

En excluant les facteurs d'attraction évidents liés au fait qu'un pays en paix se révèle attractif pour les ressortissants d'un pays en une guerre civile ou dont le caractère liberticide du régime est très net, les raisons de l'attraction, comme celles de la répulsion, sont de nature politique, économique et démographique.

D'abord, le sentiment qu'un pays dispose d'institutions ou de dirigeants impliquant l'existence d'une certaine stabilité des règles de droit ${ }^{26}$ et d'une relative concorde sociale peut le rendre attirant. C'est le cas d'un territoire comme Mayotte, dont l'État de droit et l'offre de services publics sont incontestablement supérieurs à celle des pays voisins. Autre exemple : la Côte d'Ivoire qui, pendant toute la période d'Houphouët-Boigny, se disait prête à recevoir des Africains. L'Afrique du Sud, en dépit des difficultés de sa démocratie, est considérée comme disposant d'un régime politique moins imparfait que nombre d'autres pays d'Afrique.

De son côté, le Maroc, longtemps considéré exclusivement comme un pays de transit pour des Africains subsahariens voulant rejoindre l'Europe, s'est révélé, surtout depuis le début du XXIe siècle, un pays de peuplement pour des Africains subsahariens ${ }^{27}$ non seulement face aux difficultés à gagner l'Europe, mais aussi parce que le Maroc compte des éléments politiques attractifs qu'il a décidé d'accroître. D’abord, le Maroc dispose d'une certaine stabilité institutionnelle, assez peu courante

${ }^{25}$ Dumont, Gérard-François, Kanté, Seydou, «L'émigration sénégalaise : autant Sud-Sud que SudNord », dans: Moriniaux, Vincent (direction), Les mobilités, Paris, Éditions Sedes, 2010 ; Dumont, GérardFrançois, Kanté, Seydou, La géopolitique du Sénégal. De Senghor à l'élection de Macky Sall, Paris, L'Harmattan, 2019.

${ }^{26}$ Dumont, Gérard-François, «Géopolitique des migrations : les effets répulsifs ou attractifs des Constitutions », Diploweb.com, La revue géopolitique, 26 juin 2016.

${ }^{27}$ Lahlou, Mehdi, «Le Maroc et les migrations subsahariennes », Population \& Avenir, $\mathrm{n}^{\circ} 659$, septembre-octobre 2002. 
en Afrique. Ensuite, le Maroc a pu améliorer son image auprès d'Africains subsahariens par différentes initiatives peu communes en Afrique. Ainsi, après 1990, le Maroc change-t-il d'attitude sur la question des droits humains. Le pouvoir marocain choisit d'abandonner son attitude peu respectueuse à leur égard et prend des initiatives témoignant d'un véritable changement: création du Conseil consultatif des droits de l'homme (CCDH), d'un ministère, et reconnaissance de ces droits dans le préambule de la Constitution de septembre 1992. Ces changements positifs, au niveau législatif, s'accompagnent de la libération de centaines de détenus politiques, du retour de la plupart des exilés et de la réapparition d'une bonne partie des disparus. Tout se passe comme si Hassan II tenait à améliorer le respect des droits de l'homme avant sa mort, qu'il sentait proche. En juin 2003, le Maroc se rapproche des standards européens avec le vote, le 26 juin 2003, par le Parlement marocain, de la loi n 02-03 sur l'entrée et le séjour des étrangers au Maroc. Ensuite, le roi Mohammed VI, au pouvoir à compter de 1999, installe le 12 avril 2004 une Instance équité et réconciliation dont le but est de rendre justice aux victimes des exactions commises sous le règne précédent ${ }^{28}$. En outre, l'image du Maroc se trouve améliorée dans les pays d'Afrique subsaharienne par plusieurs initiatives diplomatiques dont témoignent des visites d'État du roi Mohammed VI. Ainsi, le Royaume a enregistré deux grandes réussites diplomatiques sur le continent africain: sa réintégration au sein de l'Union africaine le 30 janvier 2017 et l'accord de principe de son adhésion à la CEDEAO les 5 et 6 juin 2017 lors du 51 ${ }^{\text {e }}$ sommet de la CEDEAO à Monrovia. Rappelons que, membre fondateur et actif de l'OUA (devenue Union africaine) en 1960, le Maroc avait quitté l'organisation en 1984 lorsque 26 des 50 États membres ont voté pour l'admission de la République sahraouie (Sahara Occidental) au sein de l'Union africaine.

Par ailleurs, pendant cinquante ans, du 7 novembre 1956, date de ratification de la Convention de Genève sur les Réfugiés (1951) par le Royaume du Maroc, jusqu'à l'automne 2004, la représentation au Maroc du Haut-commissaire aux réfugiés (HCR) de l'Organisation des Nations Unies est restée symbolique, puis cette situation a évolué.

Le 6 novembre 2013, à l'occasion de 38e anniversaire de la « marche verte », le roi prononce un discours qui vaut reconnaissance officielle de son pays comme terre d'immigration pour l'Afrique subsaharienne : "Les relations privilégiées qui unissent le Maroc aux pays de l'Afrique subsaharienne ne sont pas que politiques et économiques. Ce sont, dans le fond, des liens humains et spirituels séculaires. Eu égard à la situation qui prévaut dans certains de ces pays, nombre de leurs citoyens immigrent au Maroc d'une façon légale ou illégale. Jadis point de passage vers l'Europe, notre pays s'est transformé en destination de résidence. Face à l'accroissement sensible du nombre des immigrés venus d'Afrique ou d'Europe, Nous avons invité le gouvernement à élaborer une nouvelle politique globale relative aux questions d'immigration et d'asile, suivant une approche humanitaire conforme aux engagements internationaux de notre pays et respectueuse des droits des immigrés. Pour illustrer

${ }^{28}$ Auparavant, jusqu'en 1990, période notamment marquée par des attentats visant à le renverser et auxquels il a échappé, parfois miraculeusement, le roi Hassan II a assumé une politique se traduisant par des prisonniers politique considérés comme des criminels de droit commun, des centaines de disparus séquestrés et des Marocains contraints à l'exil. Mais le pouvoir nie alors l'existence de prisonniers politiques, ainsi que sa responsabilité dans les disparitions. 
l'intérêt particulier que Nous attachons à ce volet, Nous avons tenu à charger un département ministériel des questions d'immigration. »

Ce discours est suivi d'actes concrets. Par exemple, le 2 janvier 2014, le Maroc lance une opération de régularisation de la situation administrative des étrangers en séjour illégal au Maroc. Puis, le 18 décembre 2014, le Conseil du gouvernement adopte une "stratégie nationale de l'immigration et de l'asile ». Parallèlement, l'élaboration et la mise en place d'un système national d'asile se poursuivent.

Les facteurs politiques d'attraction ont souvent été complétés par des facteurs économiques dans la mesure où la bonne santé économique d'un pays est rarement possible sans une certaine clairvoyance politique y organisant des règles stables. Les pays politiquement les mieux dotés bénéficient également, en général, des meilleurs niveaux de vie. Par exemple, le PIB par habitant de Mayotte est certes très faible par rapport à la moyenne de l'UE, mais il est incomparablement supérieur à la moyenne de l'Afrique (4 965 US\$/habitant en parité de pouvoir d'achat) et plus encore à celui des Comores, qui n'est que de 1570 US\$ par habitant ${ }^{29}$.

L'attractivité économique peut aussi exister dans un État « rentier » bénéficiant d'importantes ressources d'hydrocarbures. C'est ainsi qu'avant sa déstabilisation en 2011, la Libye était un pays fortement attractif pour des populations africaines. C'est toujours le cas, aujourd'hui, de la Guinée équatoriale ou de l'Angola dont les soldes migratoires positifs pourraient cependant se réduire sous l'effet du prix moins élevé du baril de pétrole.

En matière d'attraction démographique, l'un des taux d'accroissement les plus élevés est celui du Sahara occidental, région dont le statut, depuis le départ des Espagnols en 1975, n'a pas fait l'objet d'accords internationaux. Le potentiel économique de cette région n'est pas négligeable, alors que son peuplement est très faible, estimé à 266000 habitants sur un territoire d'une superficie équivalant presque à la moitié de celle de la France métropolitaine. Ses apports migratoires viennent essentiellement du Maroc - ou du nord du Maroc, selon Rabat -, car le gouvernement marocain souhaite mieux asseoir sa souveraineté sur ce territoire en le peuplant.

Parmi les pays africains à taux d'accroissement migratoire positif figurent également le Gabon et le Botswana, deux pays dont le nombre d'habitants est très faible par rapport à leur potentiel économique. Aussi le Gabon attire-t-il des immigrants de très nombreux pays d'Afrique, notamment pour travailler dans ses industries pétrolières onshore ou dans ses industries forestières (bois d'okoumé et d'ozigo).

Comme partout et toujours dans le monde, les migrations internationales résultent de la combinaison de deux groupes de facteurs essentiels : des phénomènes répulsifs dus à des perspectives médiocres, voire catastrophiques dans un pays, et des phénomènes attractifs au sein de territoires offrant des opportunités, des espoirs d'amélioration ou tout simplement s'inscrivant dans un rêve de réussite. Il n'est donc pas anormal que des pays africains s'inscrivent dans ces logiques. Mais ces dernières

${ }^{29}$ Sardon, Jean-Paul, «La population des continents et des pays », Population \& Avenir, $n^{\circ} 740$, novembre-décembre 2018. 
s'y trouvent intensifiées pour deux raisons principales: la piètre gouvernance de nombreux régimes africains, incluant des pratiques de corruption, et les conditions non réalisées du développement dans des États qui restent à construire.

Les violations des droits de l'homme commises par des régimes dictatoriaux, voire totalitaires, et les nombreux conflits civils qui secouent périodiquement le continent africain multiplient les émigrations forcées. Les réfugiés, de jure ou de facto, qui forment les plus nombreux flux migratoires intra-africains, se retrouvent souvent dans des pays voisins de leur territoire d'origine ${ }^{30}$. Seule une minorité d'entre eux parvient à aller solliciter une demande d'asile politique dans les pays européens.

En outre, même lorsque des territoires ne sont pas dévastés par les conflits, l'Afrique a multiplié les cas d'école de la mauvaise gestion économique, étouffant les possibilités de création d'emploi et les espoirs de développement. L'association de l'insécurité économique et du chômage pousse alors des populations à se rendre par exemple dans les pays africains sachant mieux profiter de leur économie de rente ou plus efficients, d'où l'importance des migrations Sud-Sud vers le Gabon, la Tanzanie, le Kenya, l'Afrique du Sud et, pendant plusieurs décennies, la Côte d'Ivoire. Les malheurs politiques de ce dernier pays, dans la quinzaine d'années qui a suivi la mort d'Houphouët-Boigny, symbolisent les difficultés africaines dans l'accès au développement. Ces dernières concourent à une émigration Sud-Nord; celle-ci s'inscrivait auparavant pour l'essentiel dans une logique de proximité géographique mais a vu son champ s'étendre à tous les continents.

Le futur migratoire de l'Afrique dépend des évolutions politico-économiques. Si les spasmes de jeunesse que rencontrent certains États, le plus souvent récents, disparaissent, si les associations économiques régionales et sous-régionales parviennent à des résultats, si les efforts de partenariat du Nord réussissent, l'Afrique n'a pas de raison de rester répulsive puisque son potentiel économique est considérable. Dans le cas contraire, rien ne peut empêcher la continuation d'importants flux migratoires tant intracontinentaux qu'intercontinentaux.

G.-F.D.

\begin{tabular}{|c|}
\hline $\begin{array}{c}\text { Les analyses de Population \& Avenir } \\
\text { 35 Avenue Mac Mahon } \\
75017 \text { Paris } \\
\text { tel. (0)1 } 47705381 \text { Fax : (0)1 } 73020064 \\
\text { courriel e-mail : revuepopulationetavenir@gmail.com } \\
\text { ISSN 2552-2078 } \\
\text { Directeur de la publication : Gérard-François Dumont }\end{array}$ \\
\hline $\begin{array}{l}\text { La publication Les analyses de Population \& Avenir a vocation à contribuer au débat public sur les questions de populations } \\
\text { et de territoires. Les opinions exprimées dans ce texte n'engagent que la responsabilité des auteurs. } \\
\text { (c) Les analyses de Population \& Avenir. Droits de reproduction partielle sous réserve de conserver l'indication des sources. } \\
\text { www.population-et-avenir.com }\end{array}$ \\
\hline
\end{tabular}

\footnotetext{
${ }^{30}$ Sène, Abdourahmane Mbade, «Impacts du conflit casamançais dans la zone frontalière de la région de Ziguinchor avec la Gambie », Conférence PICARD, Malatya (Turquie), 9-11 octobre 2018.
} 


\section{La publication}

\section{Les analyses de Population \& Avenir}

a pour objet de diffuser gratuitement des analyses sur des thèmes diversifiés (fécondité, pauvreté, migration, immigration, intégration, politique familiale, logement, logement social, francophonie...), et sur des territoires variés précisés dans le titre de la publication (Europe, Union européenne, Afrique, Arabie Saoudite, Chine, France, régions françaises, Corse,...) ou étudiés au sein du thème considéré (Brésil, Maroc, Venezuela...).

Toutes Les analyses de Population \& Avenir sur: https://www.population-et-avenir.com/les-analyses-de-population-avenir/

Pour suivre la revue Population \& Avenir:

https://www.population-et-avenir.com/abonnement-annuel/abonnement-revuepopulation-avenir/

\section{Les analyses de Population \& Avenir}

Liste des publications

"La francophonie, bilan et perspectives à l'aune des rapports Graddol», Les analyses de Population \& Avenir, 2019.

«Les migrations internationales et l'Afrique : des logiques Sud-Nord ou SudSud ? », Les analyses de Population \& Avenir, 2019.

«Observations sur le Dictionnaire de démographie et des sciences de la Population », Les analyses de Population \& Avenir, 2019. 
«La reprise de la fécondité au milieu des années 1930, phénomène non perçu des observateurs du temps? », Les analyses de Population \& Avenir, 2019.

«Immigration : la question de l'intégration dans un contexte fondamentalement nouveau », Les analyses de Population \& Avenir, 2017.

«La pauvreté dans le monde : réponses inopérantes et solutions efficientes », Les analyses de Population \& Avenir, 2016.

« China : a sustainable model ?», Les analyses de Population \& Avenir, 2016.

« Chine : un modèle durable ? », Les analyses de Population \& Avenir, 2016.

«Régions françaises: des géants géographiques aux attributions minuscules », Les analyses de Population \& Avenir, décembre 2015.

"Family policies \& Europe's demographic future », Les analyses de Population \& Avenir, 2015.

«Politiques familiales et avenir démographique de l'Europe», Les analyses de Population \& Avenir, 2015.

«La « crise » des migrants, l'opération anti-passeurs « Sophia » de l'UE et l'ONU », Les analyses de Population \& Avenir, 2015.

«Europe : des disparités considérables dans les évolutions démographiques », Les analyses de Population \& Avenir, juillet 2015.

«Le logement et le logement social en France : éléments de diagnostic », Les analyses de Population \& Avenir, juin 2015.

«Territoires: un fonctionnement radial ou réticulaire?», Les analyses de Population \& Avenir, mai 2015.

«Women and «Human Rights» in Saudi Arabia », Les analyses de Population \& Avenir, septembre 2012.

«Le nouveau recensement de la population de la France et les améliorations nécessaires », Les analyses de Population \& Avenir, 2008.

«Pourquoi la Corse a-t-elle voté «non» au référendum portant sur une collectivité territoriale unique? », Les analyses de Population \& Avenir, 2003. 


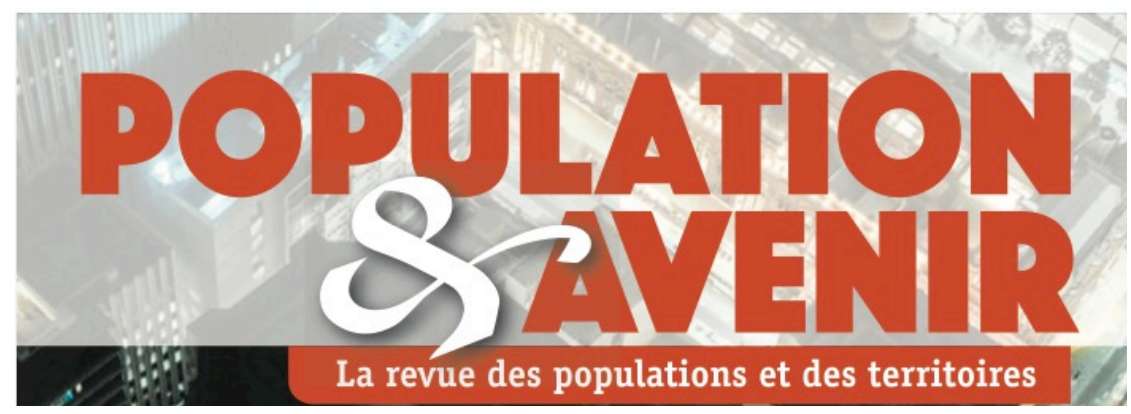

Pour suivre la revue Population \& Avenir:

https://www.population-et-avenir.com/abonnement-annuel/abonnement-revuepopulation-avenir/

Population \& Avenir est la seule revue française et européenne se consacrant exclusivement aux questions géodémographiques et à leurs impacts territoriaux, économiques et sociaux.

Son objet consiste à présenter les évolutions en France, en Europe et dans le monde sous l'éclairage révélateur de la géographie de la population et de la science démographique.

Dans ce dessein, cinq fois par an, Population \& Avenir présente clairement à ses lecteurs le privilège d'informations, d'analyses, de réflexions et d'argumentaires, illustrés par des cartes, des graphiques, des schémas...

En outre, chaque numéro de Population \& Avenir propose un exercice pédagogique, conforme aux programmes de l'enseignement, amplement illustré, et comprenant un corrigé des questions traitées. Cet exercice s'adresse aux professeurs d'histoire-géographie et de sciences économiques et sociales, ainsi qu'aux élèves et aux étudiants devant préparer des examens ou des concours. Il est également fort apprécié de tous les lecteurs qui y voient une sorte de trivial pursuit enrichissant les connaissances tout en stimulant les capacités d'analyse et de synthèse de chacun. 


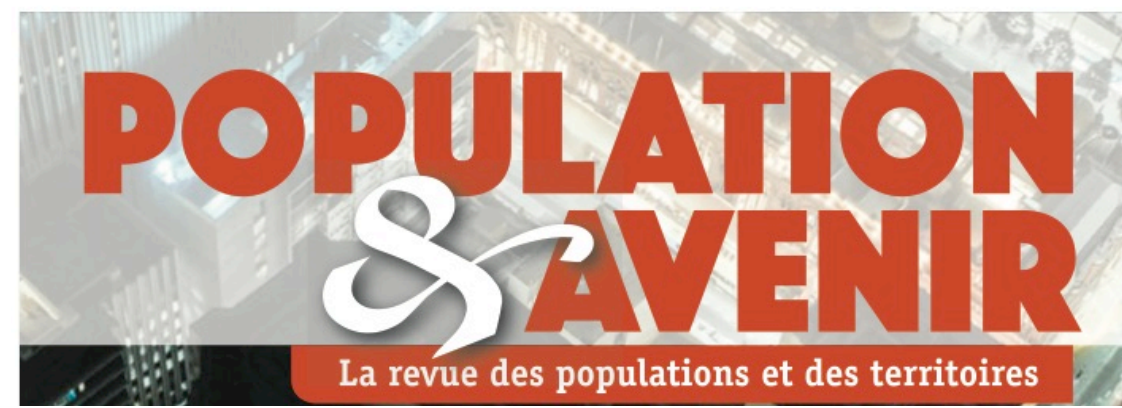

Pour suivre la revue Population \& Avenir:

https://www.population-et-avenir.com/abonnement-annuel/abonnement-revuepopulation-avenir/

La recherche d'un thème ou d'un espace géographique traité dans la revue Population \& Avenir peut s'effectuer sur les sites :

www.population-et-avenir.com

http://population-et-avenir.cairn.info

Une partie des articles et dossiers publiés dans la revue Population \& Avenir peuvent être acquis selon un format numérique sur le site de Cairn info :

https://www.cairn.info/revue-population-et-avenir.htm

Les sommaires de la revue Population \& Avenir en langue anglaise et quelques articles en anglais (english) sont accessibles à l'adresse :

https://www.cairn-int.info/journal-population-et-avenir.htm 


\section{Mieux comprendre}

les dynamiques territoriales et les évolutions géopolitiques, économiques et sociales sous l'éclairage révélateur de la démographie.

Population \& Avenir, revue indépendante alliant rigueur et pédagogie, vous présente une analyse originale des enjeux actuels. Vous y trouverez une source d'informations, de réflexions et d'argumentaires amplement illustrés par des cartes, des graphiques, des tableaux, des schémas...
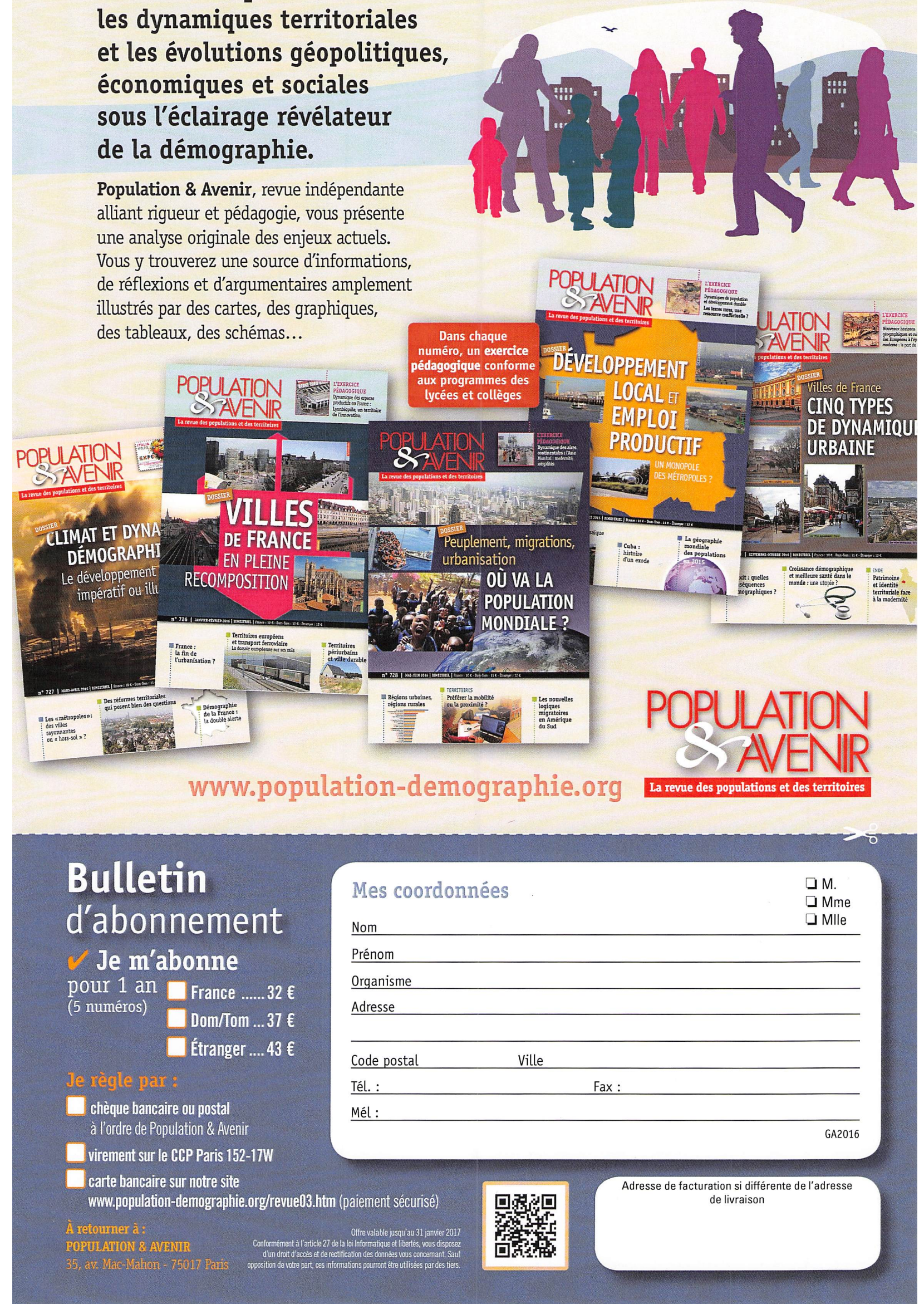